\title{
BMJ Open Association of chiropractic integration in an Ontario community health centre with prescription opioid use for chronic non-cancer pain: a mixed methods study protocol
}

\author{
Peter C Emary (D) , 1,2,3 Mark Oremus (D) , 1,4 Lawrence Mbuagbaw (D) , , ,5,6,7 \\ Jason W Busse (i) $1,8,9,10$
}

To cite: Emary PC, Oremus M, Mbuagbaw L, et al. Association of chiropractic integration in an Ontario community health centre with prescription opioid use for chronic noncancer pain: a mixed methods study protocol. BMJ Open 2021;11:e051000. doi:10.1136/ bmjopen-2021-051000

- Prepublication history and additional supplemental material for this paper are available online. To view these files, please visit the journal online (http://dx.doi.org/10.1136/ bmjopen-2021-051000).

Received 06 March 2021 Accepted 19 0ctober 2021

Check for updates

(c) Author(s) (or their employer(s)) 2021. Re-use permitted under CC BY-NC. No commercial re-use. See rights and permissions. Published by BMJ.

For numbered affiliations see end of article.

Correspondence to

Dr Peter C Emary;

emaryp@mcmaster.ca

\section{ABSTRACT}

Introduction Emerging evidence from a number of primary care centres suggests that integration of chiropractic services into chronic pain management is associated with improved clinical outcomes and high patient satisfaction as well as with reductions in physician visits, specialist referrals use of advanced imaging and prescribing of analgesics. However, formal assessments of the integration of chiropractic services into primary care settings are sparse, and the impact of such integration on prescription opioid use in chronic pain management remains uncertain. To help address this knowledge gap, we will conduct a mixed methods health service evaluation of an integrated chiropractic back pain programme in an urban community health centre in Ontario, Canada. This centre provides services to vulnerable populations with high unemployment rates, multiple comorbidities and musculoskeletal disorders that are commonly managed with prescription opioids.

Methods and analysis We will use a sequential explanatory mixed methods design, which consists of a quantitative phase followed by a qualitative phase. In the quantitative phase, we will conduct a retrospective chart review and evaluate whether receipt of chiropractic services is associated with reduced opioid use among patients already prescribed opioid therapy for chronic pain. We will measure opioid prescriptions (ie, opioid fills, number of refills and dosages) by reviewing electronic medical records of recipients and non-recipients of chiropractic services between 1 January 2014 and 31 December 2020 and use multivariable regression analysis to examine the association. In the qualitative phase, we will conduct in-depth, one-on-one interviews of patients and their general practitioners to explore perceptions of chiropractic integration and its impact on opioid use.

Ethics and dissemination This study was approved by the Hamilton Integrated Research Ethics Board at McMaster University (approval number 2021-10930). The results will be disseminated via peer-reviewed publications, conference presentations and in-person or webinar presentations to community members and healthcare professionals.
Strengths and limitations of this study

- We will link electronic medical records with medical drug claims data from the Institute for Clinical Evaluative Sciences to validate patient opioid prescriptions and dosages.

- Generalised estimating equations will be used to account for hierarchical clustering and to control for differences in confounding factors between our exposure and comparison groups.

- For our regression models, we have prespecified the anticipated direction of association for each independent variable to provide additional reassurance that associations are unlikely to be spurious if detected.

- The qualitative component of our study will provide a richer understanding of chiropractic integration and its impact on opioid prescribing.

- Causation cannot be established with our study design, and the results may not be applicable to settings outside of Canadian community health centres.

\section{INTRODUCTION}

Chronic pain is a common condition that affects approximately $20 \%$ of the global population. ${ }^{1-4}$ In North America, opioids are commonly prescribed to relieve chronic pain and improve function ${ }^{5}$; however, opioids provide only modest benefits ${ }^{6}$ and are associated with important dose-dependent harms, including overdose and death. ${ }^{7-10}$ Accordingly, the chiropractic profession has called on governments to improve support for non-opioid approaches to managing chronic pain, particularly in vulnerable and marginalised populations. ${ }^{11}$

Since 2007, a growing number of primary care centres in Canada have reported on their experiences with integration of chiropractic services into chronic pain management. ${ }^{12-19}$ Findings from these reports describe 
improvements in pain intensity and disability and high levels of patient satisfaction; additional outcomes include reductions in physician visits, specialist referrals, advanced imaging and prescribing of opioid and non-opioid analgesics. ${ }^{12-19}$ However, these studies ${ }^{12-19}$ employed single cohort, pre-post designs (ie, no comparison groups), thereby preventing a formal assessment of whether chiropractic integration improved chronic pain management compared with standard practice. ${ }^{20}$ Comparative assessments of the integration of chiropractic services into primary care settings are sparse, ${ }^{21} 22$ and the impact of such integration on prescription opioid use in chronic pain management remains uncertain.

Unanswered questions also exist about specific types of integration, namely whether the impact of chiropractic services on opioid use in patients who start with chiropractic care before being prescribed opioids differs from the impact of chiropractic care on opioid use when added later to patient management. For instance, recent evidence from three studies suggests opioid-naive patients with spine-related pain who receive chiropractic services as either standalone, or part of, first-line treatment are less likely to receive opioid prescriptions in the management of their conditions. ${ }^{23-25}$ In a 2020 systematic review and meta-analysis of six uncontrolled cohort studies, ${ }^{23}$ Corcoran et al found that patients with acute or chronic non-cancer back or neck pain who received chiropractic services were $64 \%$ less likely than non-chiropractic users to be prescribed opioid medications (pooled $\mathrm{OR}=0.36$, $95 \%$ CI 0.30 to 0.43 ). Similar findings have recently been reported by others ${ }^{24}$; however, few studies have examined whether the receipt of chiropractic services can reduce opioid use in patients with existing opioid prescriptions. Similarly, few studies have examined the association between chiropractic services and opioid use among patients with chronic, non-cancer pain (CNCP) in a community health centre (CHC) setting, and none has done so using a mixed methods approach.

\section{Aim}

To help address these knowledge gaps, we will conduct a mixed methods evaluation of a chiropractic back pain programme integrated into standard physician care at the Langs $\mathrm{CHC}^{26}$ in Cambridge, Ontario, Canada. Cambridge is a medium-sized urban municipality (population: 130000) located $82 \mathrm{~km}$ southwest of Toronto. CHCs provide services to communities and vulnerable populations with high unemployment rates, multiple comorbidities and high rates of musculoskeletal disorders and opioid use. ${ }^{12-15}$ 17-19 222627 Since chiropractic services are not publicly funded in Canada, these populations have traditionally faced barriers to accessing such services. ${ }^{12-15} 17-19$

\section{Conceptual framework}

There are several reasons why the utilisation of chiropractic services might lead to reduced opioid use in patients with CNCP. $^{131516181923-25}$ First, chiropractic care, including spinal manipulation, is efficacious in managing back and neck pain. ${ }^{28-30}$ Patients obtaining pain relief through chiropractic treatment might, in collaboration with their general practitioners (GPs), choose to taper opioid prescriptions. GPs might also prescribe fewer opioid medications or choose lower dosages if they can refer patients to chiropractors as a first-line treatment for pain management. In addition, patients accessing chiropractic services may be more resistant to using opioids than non-chiropractic patients and be more likely to seek out non-pharmacological pain management alternatives.

Our conceptual framework (online supplemental appendix 1) recognises that chronic spinal pain and opioid use can be associated with various comorbidities (eg, cardiovascular disease,${ }^{31}$ diabetes,${ }^{32}$ obesity,${ }^{33}$ depression, ${ }^{32-38}$ anxiety, ${ }^{32-37}$ somatisation $^{35}$ or poor general health ${ }^{34} 39$ as well as clinical and sociodemographic characteristics like pain severity/chronicity, ${ }^{33} 38$ smoking status, ${ }^{34} 38$ age $^{39}$ and gender. ${ }^{38}{ }^{39}$ As such, these factors could also impact patients' utilisation of chiropractic services or opioid medications. For instance, opioid overuse/misuse has been frequently reported among individuals of low socioeconomic status and those with behavioural health problems. ${ }^{36}{ }^{37}$ Chronic pain is prevalent among these groups, ${ }^{31-39}$ and a primary motivation of chronic pain sufferers for misusing opioids is to relieve physical pain. ${ }^{37}$ Therefore, our regression analyses described below ('quantitative data analysis') will control for these variables.

\section{METHODS AND ANALYSIS Reporting}

We have reported our study protocol in accordance with the Good Reporting of A Mixed Methods Study guidelines. ${ }^{40}$ We will report the results of our mixed methods manuscript in accordance with these guidelines, as well.

\section{Research questions \\ Quantitative}

In adult patients (age $\geq 18$ years) presenting with $\mathrm{CNCP}$ at the Langs CHC, does adding chiropractic care to ongoing usual medical care, compared with ongoing usual medical care alone, reduce the rate of utilisation of opioid medications for up to 12 months following the index chiropractic visit?

\section{Qualitative}

How do patients and GPs at Langs perceive the integration of chiropractic services, and do they perceive this integration has impacted opioid use among persons with CNCP? For the purposes of this study, GPs will include family physicians and nurse practitioners.

\section{Mixed methods}

In what ways do the qualitative data help explain the quantitative results? For example, if an association is found between chiropractic integration at the Langs CHC and 
opioid use, was it because the integration of chiropractic services allowed GPs to prescribe fewer opioids, were patients able to reduce their opioid intake because of improved pain management with access to these services or was chiropractic care implemented as part of a broader opioid-reducing strategy at the centre?

\section{Study design}

We will use a sequential explanatory mixed methods design. ${ }^{41}$ Data for the quantitative portion of the study will be obtained via chart review ${ }^{42}$ of electronic medical records (EMRs) of both recipients and non-recipients of chiropractic services with at least one prescribed opioid for the treatment of a CNCP-related diagnosis at the Langs CHC. We will record opioid prescription data and other sample characteristics (eg, age, sex, smoking status, body mass index (BMI), healthcare visit frequency and comorbidities such as depression, anxiety, fibromyalgia, diabetes and cardiovascular disease) for all patients and tabulate the differences in opioid prescriptions (ie, opioid fills, number of refills and dosages) between the two groups. We will use multivariable regression analysis to examine whether the receipt of chiropractic services is associated with a reduction in the number or dose of opioid prescriptions. For the qualitative portion of the study, we will conduct in-depth, one-on-one interviews of patients and GPs from the Langs CHC to further explore perceptions regarding chiropractic integration and its impact on opioid prescribing. We will use pragmatism ${ }^{4143}$ as the philosophical stance for mixing quantitative and qualitative methodologies. With this approach, the focus is on combining methods of data collection for "what works' best in answering the research question. ${ }^{41}$

\section{Rationale for mixed methods design}

Our rationale for using a mixed methods design will be that of complementarity, ${ }^{44}$ that is, the results from the qualitative portion of the study will be used to help clarify and explain the quantitative findings. The added value of mixed methods research is that multiple methods of data collection and analysis can be employed to answer questions with a greater breadth and depth of understanding than would be possible with only one methodology alone ${ }^{4143}$ As such, the interview component of our study will allow for a more complete understanding of the barriers and facilitators to incorporating chiropractic services as well as whether these services were used by patients or GPs to reduce reliance on opioid prescribing for CNCP. The Langs $\mathrm{CHC}^{26}$ was chosen as the setting for this study because chronic pain patients and those of low socioeconomic status tend to be frequent users of healthcare services and have high rates of opioid use in general. ${ }^{32} 343637$ An illustrative diagram outlining our study procedures is provided in figure 1 .

\section{Chiropractic CHC programme}

The chiropractic programme at the Langs CHC has been described in detail elsewhere. ${ }^{1518}$ Briefly, this is a partially
Phase

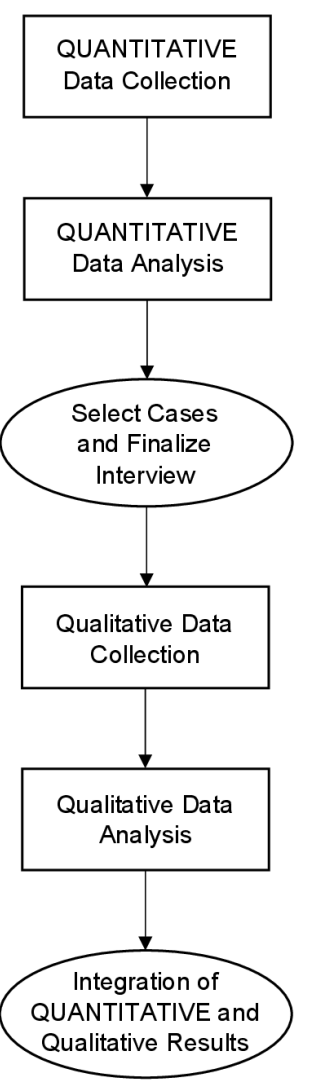

Procedure

- Retrospective chart review of opioid prescriptions for chronic non-cancer pain among recipients and nonrecipients of chiropractic services

- Descriptive statistics

- Inferential statistics (univariate and multivariable regression analysis)

- SPSS software, v26.0

- Purposive selection of patients and general practitioners from the Langs Community Health Centre based on maximum variation

- Develop interview questions

- Individual in-depth interviews $(n=6$ 10 per group, or until data saturation) - Audio recordings and field notes - Begin reflexive journaling and audit trail

- Coding and content analysis - Identify themes and subthemes

- Summarize content by thematic group with representative quotes - MAXQDA qualitative software

- Interpretation and explanation of the quantitative and qualitative findings

- Use of joint display tables and narrative weaving of results

Figure 1 Study diagram of an explanatory sequential design of a mixed methods study on the association of chiropractic integration with opioid use for chronic non-cancer pain at the Langs Community Health Centre. The quantitative and qualitative data collection and analysis phases are shown along the left side of the diagram in rectangular boxes. The two points of interface (or mixing) of the quantitative and qualitative phases are shown and described in the ovals. The term 'QUANTITATIVE' is capitalised to indicate prioritisation of the quantitative phase in the study. The study procedures for each phase are listed along the right side of the diagram. MAXQDA, Max Weber Qualitative Data Analysis; SPSS, Statistical Package for the Social Sciences.

subsidised, complimentary service that operates on two separate days of the week (for a total of 4 hours per week) and is provided on a rotating basis by a team of between three to four chiropractors. The service is only accessible to patients registered at the $\mathrm{CHC}$ and through referral from their GP. Treatment sessions are evidence based ${ }^{45}$ and include spinal manipulation, soft-tissue therapy, education, reassurance and home advice (eg, icing, stretching and strengthening exercises). Patients are typically discharged from care after 3 months or a maximum of 12 visits; however, some continue with treatment to address episodes of exacerbation/flare-up or chronic pain. Patients also continue to receive usual medical care at the $\mathrm{CHC}$, such as GP assessment, prescription medication and referral for diagnostic testing or specialist consultation as well as other cointerventions (eg, visits 
Table 1 Summary of variables to be used for the quantitative analysis

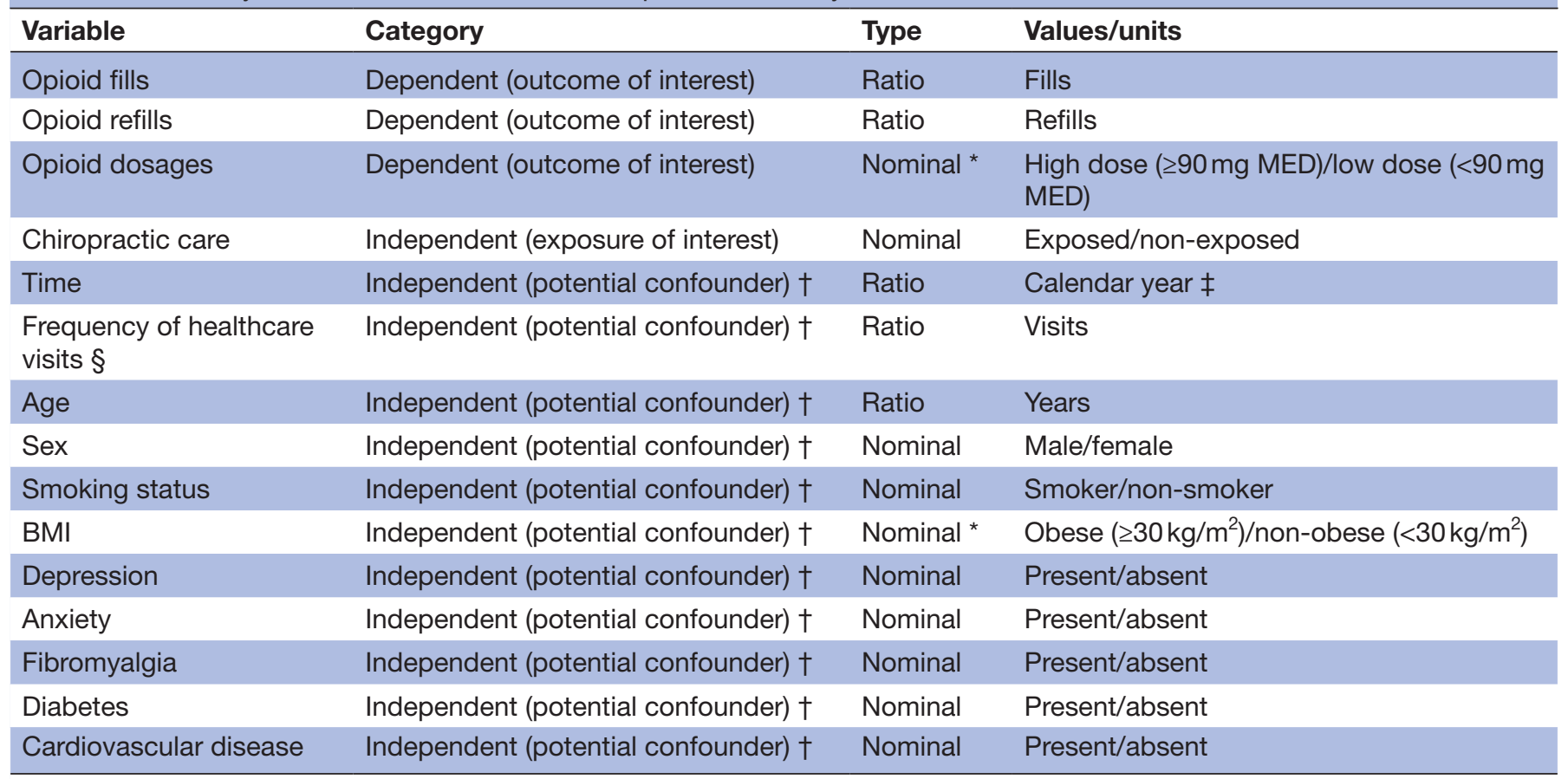

*Opioid dosages and BMI will be dichotomised from continuous variables for comparative analysis.

†Data from the earlier years in the project's 7-year timeframe, a higher frequency of healthcare visits, younger age, male sex, smoking, obesity, depression, anxiety, fibromyalgia, diabetes and cardiovascular disease are anticipated to be positively associated with opioid use. $\ddagger$ Calendar years will be measured at the patient’s index visit date to the CHC for a CNCP-related diagnosis. $\S$ Healthcare visits will constitute general practitioner and chiropractic visits.

$\mathrm{BMI}$, body mass index; $\mathrm{CHC}$, community health centre; CNCP, chronic non-cancer pain; MED, morphine equivalents daily.

with nurses, dieticians, social workers or physical/massage therapists) as required.

\section{Quantitative sampling}

For the quantitative phase, we will include records from adult patients, aged $\geq 18$ years with one or more active prescriptions of opioid medication and who presented with CNCP (pain of $\geq 12$ weeks' duration, not associated with a diagnosis of cancer) of the back or neck at the Langs CHC between 1 January 2014 and 31 December 2020. The start date for quantitative sampling was chosen as 1 January 2014 because this was the inaugural date of the CHC's chiropractic programme. ${ }^{15}$ Patients with spinal neoplasms or other contraindications to chiropractic treatment (ie, 'red flag' diagnoses such as fractures, infections, inflammatory arthritis or cauda equina syndrome) will be excluded. We will randomly select an equal number of recipients and non-recipients of chiropractic services within the aforementioned 7-year timeframe to form our exposure and comparison cohorts. Our sampling frame will be the Langs EMR database, and our sampling unit will be individual patient records. To guard against overfitting of our regression models, ${ }^{46}$ we will set a minimum threshold of 10 events per category for each independent variable (see variable categories in table 1 ) to ensure that each variable has sufficient discriminant power to detect an association with opioid use, if an association exists. Based on patient recruitment numbers from our pilot study, ${ }^{15}$ we expect to be able to select the sample from approximately 500 to 750 eligible patient charts.

Rates of missing data involving EMR extraction can vary. ${ }^{47}$ Therefore, for validation (ie, to verify patient opioid prescriptions and dosages), the final set of EMR records will be linked with medical drug claims data from the Narcotics Monitoring System and Ontario Drug Benefit Program databases. We have confirmation from the Data and Analytic Services Department at the Institute for Clinical Evaluative Sciences (ICES) (https://www. ices.on.ca) that we will have access to the claims information (approval number 2020-739). ICES is an independent, non-profit research organisation that maintains a data repository of publicly funded administrative health service records for all Canadian citizens in the province of Ontario.

\section{Quantitative data collection}

Our outcome variables will be measured as the number of prescribed opioid fills, the number of prescribed opioid refills and the prescribed opioid dosages among patients in the exposure and comparison groups. These outcomes will be measured in each patient chart from both groups up to 12 months after their index visit for a CNCP-related diagnosis of the back or neck at the CHC. Although we will only extract data for patient encounters related to a back or neck pain diagnosis including opioid medications prescribed on these visit dates, it remains possible 
that opioids may have been prescribed for other indications. This may attenuate the association between chiropractic care and opioid use. ${ }^{48}$ Other variables that will be extracted from the EMR include sociodemographics (age and sex), general health (smoking status and BMI), comorbidities (depression, anxiety, fibromyalgia, diabetes and cardiovascular disease) and the total number of healthcare (ie, GP or chiropractic) visits. These variables have been shown to be associated with CNCP and opioid use. ${ }^{31-39}$ In our analysis, we will explore variance inflation factors (VIFs) to assess collinearity between independent variables. If multicollinearity is detected between two or more variables (ie, VIFs $\geq 10$ ), ${ }^{49}$ then we will compare regression models, each separately containing one of the collinear variables, to one another and select the model containing the variable that produces the lowest Akaike information criterion. Complete lists of study variables and diagnostic codes are given in table 1 and online supplemental appendix 2, respectively.

To increase the reliability of data extraction, ${ }^{42}$ two extractors will independently extract data from each chart and resolve differences by consensus. The principal investigator (PI) will adjudicate if necessary. The PI will also train and monitor the extractors, who will be blinded to the research questions. The PI will create standardised forms and a procedural manual to guide training and extraction. The extractors will pilot test the forms on a random sample of patient charts (eg, 10 from each of the exposure and comparison groups), and inter-rater agreement will be measured using the kappa $(\kappa)$ statistic.

\section{Quantitative data analysis}

Baseline characteristics will be compared between the exposed and non-exposed groups using the $\chi^{2}$ and independent $t$ tests (or Fisher exact and Wilcoxon-MannWhitney tests when appropriate) for categorical and continuous variables, respectively. Generalised estimating equations (GEEs) will be used to explore the associations between exposure to chiropractic care and opioid prescribing. ${ }^{50} 51$ To account for potential data clustering within-subjects or between medical or chiropractic practitioners, a robust variance estimator will be used to compute the standard errors for the coefficient estimates. In addition, because we do not know the structure of this correlation, we will conduct a sensitivity analysis and run a series of models testing different working correlation structures, including independent, autoregressive and unstructured matrices. ${ }^{50}{ }^{51}$ The specified link function in our GEE models will be based on the data distribution (eg, log-linear for Poisson, binomial for binary data).

GEEs with a Poisson distribution will be used when the outcomes are counts (ie, total number of opioid fills and refills over the entire course of follow-up, tabulated at the end of follow-up). Incidence density (rate) ratios for differences between the chiropractic and nonchiropractic groups will be estimated using Poisson loglinear GEEs and reported with $95 \%$ CIs and $p$ values.
A repeated-measure GEE with a binomial distribution will be used when the outcome is opioid dosage. Opioid dosages will be assessed at 90-day intervals and dichotomised into high $(\geq 90 \mathrm{mg}$ ) morphine equivalents daily (MED) or low $(<90 \mathrm{mg}) \mathrm{MED}^{6}$ and compared between the chiropractic and non-chiropractic groups from baseline to 12-month follow-up. We will estimate betweengroup differences for dosage using a binary logistic GEE and report with ORs, $95 \%$ CIs and $p$ values. To calculate the MED for each prescribed opioid, we will multiply the quantity $\times$ the milligrams per unit dispensed $\times$ drug-specific conversion factors (online supplementary appendix 3) ${ }^{68}$

For each outcome of interest, univariate and multivariable models will be built to estimate the crude and adjusted associations, respectively, between patients with and without chiropractic integration ( 1 =with; $0=$ without) and the outcomes that were described above. Covariates will be grouped into blocks (ie, sociodemographic, health-related, depressive symptoms, health behaviours and healthcare visits) and sequentially entered into the models, with time (ie, calendar year) as an additional covariate and chiropractic/non-chiropractic as the main exposure variable. Based on the previous literature, ${ }^{831-39}$ we anticipate that younger age, male sex, health-related comorbidities, depressive symptoms, poor health behaviours (eg, smoking), a higher frequency of healthcare provider visits and earlier years of our 7-year project timeframe will be positively associated with opioid use. We will report all models and assess model fit using the quasi-likelihood under the independence model criterion (QIC). ${ }^{51}{ }^{52}$ Correlation structures with the lowest QIC scores (closest to zero) will be judged as the best model fit for the data. All data and comparative analyses will be performed using SPSS V.26.0 (IBM SPSS Statistics), and the two-sided statistical significance level $(\alpha)$ for all quantitative analyses will be $5 \%$.

\section{Qualitative sampling}

For the qualitative phase, we will use stratified purposive sampling to select a subsample of patients to participate in one-on-one interviews. ${ }^{53}$ To maintain privacy of individuals' medical records, these patients will be chosen by their GPs and will be selected from among the sample whose charts we examined in the quantitative phase. This will be the first stage of integration (ie, connecting and building ${ }^{54}$ ) between the quantitative and qualitative phases of our study. We will also recruit a purposive sample of GPs from the CHC. Maximum variation ${ }^{53}$ will be used in choosing patients and GPs to ensure a range of perspectives and sociodemographic characteristics. For example, we will ask GPs to select chiropractic and nonchiropractic patients from three groups: persons whose number of opioid prescriptions has decreased over time; persons whose opioid prescriptions have remained stable or persons whose prescriptions have increased. We will provide GPs with participant recruitment guidelines. We will also recruit both medical doctors and nurse practitioners to participate in the GPs' interviews. We will select 
a minimum of 6-10 GPs and 12-20 patients (including an equal representation of chiropractic and non-chiropractic patients) on a rolling basis. ${ }^{53}$ Recruitment will cease when we reach data saturation across subsamples (ie, the point at which no new information is obtained from participants in the GP, chiropractic and non-chiropractic groups) ${ }^{55}$ We anticipate that this will happen after a total of 20-25 interviews.

\section{Qualitative data collection}

Qualitative data will be collected through the use of one-on-one (individual) semistructured interviews and framed within the principles of qualitative description. ${ }^{55}$ Based on the findings from our quantitative analysis and relevant literature, ${ }^{12-19} 22$ we will develop interview guides for both patients and GPs, which will include open-ended questions asking participants about their perceptions of the integration of chiropractic services at the Langs CHC as well as how they feel these services have impacted opioid use for chronic pain. We are aware that the impact of chiropractic care on opioid use might have been affected by whether or not GPs engaged patients in a formal effort to taper their opioid prescriptions, and if patients agreed to subsequently engage in opioid tapering. ${ }^{56} \mathrm{In}$ addition, some patients may have engaged in other nonpharmacological pain management practices outside the CHC. Those with prior positive experience with chiropractic treatment may also have been more open to being referred by their GP for these services. As such, we will explore these issues as part of the interview process. We will also collect demographic and clinical characteristic data from interview participants, including age, sex, years in practice (for GPs) or years attending the CHC (for patients), primary pain complaint and current opioid dose.

We expect each interview to last approximately 1 hour; all interviews will be audio recorded. Field notes will be taken during the interviews to document emergent themes and other observations. To promote rigour, we will triangulate source data ${ }^{41}$ by comparing themes and subthemes generated from both the patient and GP interviews. This will help facilitate a deeper understanding of the qualitative findings. ${ }^{41}$ Trustworthiness of our qualitative data will be further assessed via member checking ${ }^{41}$ (ie, participants will be sent raw transcripts of their interviews as well as a summary of the major themes to be reviewed and affirmed). In addition, a reflexive journal and audit trail will be kept to track potential researcher bias and to document decisions made around qualitative data collection and analysis procedures. ${ }^{55}$

\section{Qualitative data analysis}

All interview audio recordings will be transferred into the software programme, Max Weber Qualitative Data Analysis (MAXQDA) (http://www.maxqda.com) and transcribed verbatim. Participant identifiers will be removed. All transcripts will be independently coded by two investigators into themes and subthemes using an inductive content analytic approach. ${ }^{54}$ The investigators will meet after every five interviews ${ }^{57}$ to compare their themes and arrive at a final, agreed-upon set of themes through discussion. These themes will be organised into tabular form and the investigators will select representative quotations for each theme/subtheme. ${ }^{41}$ As part of our data integration procedures (see figure 1), we will create joint display tables ${ }^{4154}$ by adding a column for quotes to the tables reporting the regression models. In addition, we will integrate our qualitative and quantitative results using a weaving narrative approach (ie, the quantitative and qualitative results will be organised and presented side-by-side and discussed in terms of how they are similar or dissimilar) ${ }^{4154}$ As such, our follow-up qualitative findings will be used to illuminate the statistical findings from the initial quantitative chart review. ${ }^{41}$ We will then draw on the qualitative and quantitative results jointly to come to a set of conclusions (ie, 'meta-inferences'). ${ }^{435}$

\section{Patient and public involvement}

Patients and the public were not involved at this stage of the project.

\section{ETHICS AND DISSEMINATION}

This study has been approved by the Hamilton Integrated Research Ethics Board at McMaster University (approval number 2021-10930). Approval to conduct this project has also been obtained from the Chief Executive Officer at the Langs CHC. ${ }^{26}$

An important consideration for the EMR review will be confidentiality of patient records. ${ }^{42}$ To address this risk, we will have an administrator at the Langs CHC generate a list of unique identification numbers for the randomly selected patient files. The data extractors will review these files through the EMR's 'Cumulative Patient Profile' page (see, eg, online supplemental appendix 4) and enter relevant data into Microsoft Excel. Furthermore, we will only report aggregate data in any publications.

Informed consent will be obtained from interview participants and the transcripts of their interviews will be deidentified prior to analysis. All chart review data will also be recorded anonymously, deidentified and coded. Audio recordings, transcripts and extracted chart data will be stored securely on a password-protected server at the Langs CHC. Any written paper records will be stored in the PI's locked private office. All data will be destroyed after 10 years.

Dissemination of our study will occur via three peerreviewed publications (ie, the study protocol, quantitative results and a mixed methods paper) as well as conference presentations. We will also provide written summaries and oral presentations to stakeholders (ie, patients, healthcare providers, decision-makers and community members) at the Langs CHC. We will establish connections with other stakeholder groups (eg, the Canadian Pain Society (https://www.canadianpainsociety.ca)) and 
present our findings to them, either in-person or via webinars.

\section{Author affiliations}

${ }^{1}$ Health Research Methods, Evidence and Impact, McMaster University, Hamilton, Ontario, Canada

${ }^{2}$ Chiropractic, D'Youville College, Buffalo, New York, USA

${ }^{3}$ School of Public Health Sciences, Private Practice, Cambridge, Ontario, Canada ${ }^{4}$ School of Public Health Sciences, University of Waterloo, Waterloo, Ontario, Canada ${ }^{5}$ Biostatistics Unit, Father Sean 0'Sullivan Research Centre, St. Joseph's Healthcare, Hamilton, Ontario, Canada

${ }^{6}$ Centre for Development of Best Practices in Health (CDBPH), Yaoundé Central Hospital, Yaoundé, Cameroon

${ }^{7}$ Global Health, Stellenbosch University, Cape Town, South Africa

${ }^{8}$ Anesthesia, McMaster University, Hamilton, Ontario, Canada

${ }^{9}$ Michael G. DeGroote National Pain Centre, McMaster University, Hamilton, Ontario, Canada

${ }^{10}$ Chronic Pain Centre of Excellence for Canadian Veterans, Hamilton, Ontario, Canada

\section{Twitter Jason W Busse @JasonWBusse}

Acknowledgements The corresponding author thanks Dr. Behnam Sadeghirad, PharmD, MPH, PhD for helpful discussion and statistical advice.

Contributors Concept development: PCE; design: PCE, MO, LM, JWB; supervision: MO, LM, JWB; methods consultation: MO, LM, JWB; literature search: PCE; writing of protocol manuscript: PCE; critical review of protocol manuscript for intellectual content: PCE, MO, LM, JWB. All authors read and approved the final manuscript.

Funding This work was supported by the Canadian Chiropractic Research Foundation (grant number 2020-03).

Competing interests PCE is supported by research grants from McMaster University and the NCMIC Foundation for graduate studies outside of the submitted work.

\section{Patient consent for publication Not applicable.}

Ethics approval Ethics approval was obtained from the Hamilton Integrated Research Ethics Board at McMaster University (approval number 2021-10930).

Provenance and peer review Not commissioned; externally peer reviewed.

Supplemental material This content has been supplied by the author(s). It has not been vetted by BMJ Publishing Group Limited (BMJ) and may not have been peer-reviewed. Any opinions or recommendations discussed are solely those of the author(s) and are not endorsed by BMJ. BMJ disclaims all liability and responsibility arising from any reliance placed on the content. Where the content includes any translated material, BMJ does not warrant the accuracy and reliability of the translations (including but not limited to local regulations, clinical guidelines, terminology, drug names and drug dosages), and is not responsible for any error and/or omissions arising from translation and adaptation or otherwise.

Open access This is an open access article distributed in accordance with the Creative Commons Attribution Non Commercial (CC BY-NC 4.0) license, which permits others to distribute, remix, adapt, build upon this work non-commercially, and license their derivative works on different terms, provided the original work is properly cited, appropriate credit is given, any changes made indicated, and the use is non-commercial. See: http://creativecommons.org/licenses/by-nc/4.0/.

\section{ORCID iDs}

Peter C Emary http://orcid.org/0000-0003-3044-8415

Mark Oremus http://orcid.org/0000-0001-8190-253X

Lawrence Mbuagbaw http://orcid.org/0000-0001-5855-5461

Jason W Busse http://orcid.org/0000-0002-0178-8712

\section{REFERENCES}

1 Dahlhamer J, Lucas J, Zelaya C, et al. Prevalence of chronic pain and high-impact chronic pain among adults - United States, 2016. MMWR Morb Mortal Wkly Rep 2018;67:1001-6.

2 van Hecke O, Torrance N, Smith BH. Chronic pain epidemiology and its clinical relevance. Br J Anaesth 2013;111:13-18.

3 Breivik H, Collett B, Ventafridda V, et al. Survey of chronic pain in Europe: prevalence, impact on daily life, and treatment. Eur J Pain 2006;10:287-333.
4 Sá KN, Moreira L, Baptista AF, et al. Prevalence of chronic pain in developing countries: systematic review and meta-analysis. Pain Rep 2019;4:e779.

5 International Narcotics Control Board. Narcotic drugs: estimated world requirements for 2020, 2009. Available: https://www.incb.org/ documents/Narcotic-Drugs/Technical-Publications/2019/Narcotic_ Drugs_Technical_Publication_2019_web.pdf [Accessed 08 Dec 2020].

6 Busse JW, Wang L, Kamaleldin M, et al. Opioids for chronic noncancer pain: a systematic review and meta-analysis. JAMA 2018;320:2448-60.

7 Gomes T, Greaves S, Martins D. Latest trends in opioid-related deaths in Ontario: 1991 to 2015. Toronto: Ontario Drug Policy Research Network, 2017.

8 Busse JW, Craigie S, Juurlink DN, et al. Guideline for opioid therapy and chronic noncancer pain. CMAJ 2017;189:E659-66.

9 Bedson J, Chen Y, Ashworth J, et al. Risk of adverse events in patients prescribed long-term opioids: a cohort study in the UK clinical practice research Datalink. Eur J Pain 2019;23:908-22.

10 Gwira Baumblatt JA, Wiedeman C, Dunn JR, et al. High-risk use by patients prescribed opioids for pain and its role in overdose deaths. JAMA Intern Med 2014;174:796-801.

11 Canadian Chiropractic Association. A Better Approach to Pain Management: Responding to Canada's Opioid Crisis, 2016. Available: https://www.chiropractic.ca/wp-content/uploads/2016/ 11/A-Better-Approach-to-Pain-Management-in-Canada3-1.pdf [Accessed 01 Mar 2021].

12 Garner MJ, Aker P, Balon J, et al. Chiropractic care of musculoskeletal disorders in a unique population within Canadian community health centers. $J$ Manipulative Physiol Ther 2007;30:165-70.

13 Mior S, Gamble B, Barnsley J, et al. Changes in primary care physician's management of low back pain in a model of interprofessional collaborative care: an uncontrolled before-after study. Chiropr Man Therap 2013;21:6.

14 Passmore SR, Toth A, Kanovsky J, et al. Initial integration of chiropractic services into a provincially funded inner city community health centre: a program description. J Can Chiropr Assoc 2015;59:363-72.

15 Emary PC, Brown AL, Cameron DF, et al. Management of back pain-related disorders in a community with limited access to health care services: a description of integration of chiropractors as service providers. J Manipulative Physiol Ther 2017;40:635-42.

16 Centre for Effective Practice. Primary care low back pain pilot evaluation. final report. Toronto: Centre for Effective Practice, 2017.

17 Manansala C, Passmore S, Pohlman K, et al. Change in young people's spine pain following chiropractic care at a publicly funded healthcare facility in Canada. Complement Ther Clin Pract 2019;35:301-7.

18 Emary PC, Brown AL, Cameron DF, et al. Chiropractic integration within a community health centre: a cost description and partial analysis of cost-utility from the perspective of the institution. $J$ Can Chiropr Assoc 2019;63:64-79.

19 Passmore S, Manansala C, Malone Q, et al. 162. Opioid usage patterns, patient characteristics, and the role of chiropractic services in a publicly funded inner city health care facility. Spine $J$ 2019;19:S78-9.

20 Cook PF. Study designs for program evaluation: how do we know what works? J Spec Pediatr Nurs 2009;14:70-2.

21 Goertz CM, Long CR, Vining RD, et al. Effect of usual medical care plus chiropractic care vs usual medical care alone on pain and disability among US service members with low back pain: a comparative effectiveness clinical trial. JAMA Netw Open 2018;1:e180105.

22 Prater C, Tepe M, Battaglia P. Integrating a multidisciplinary pain team and chiropractic care in a community health center: an observational study of managing chronic spinal pain. J Prim Care Community Health 2020;11:215013272095368.

23 Corcoran KL, Bastian LA, Gunderson CG, et al. Association between chiropractic use and opioid receipt among patients with spinal pain: a systematic review and meta-analysis. Pain Med 2020;21:e139-45.

24 Kazis LE, Ameli O, Rothendler J, et al. Observational retrospective study of the association of initial healthcare provider for new-onset low back pain with early and long-term opioid use. BMJ Open 2019;9:e028633.

25 Whedon JM, Toler AWJ, Kazal LA, et al. Impact of chiropractic care on use of prescription opioids in patients with spinal pain. Pain Med 2020;21:3567-73.

26 Langs. Cambridge: Langs Community Health Centre, 2018. Available: https://www.langs.org [Accessed 20 Apr 2020]. 
27 Community Health Centres. Toronto: Ontario Ministry of Health and Long-Term Care, 2020. Available: https://www.ontario.ca/page/ community-health-centres [Accessed 20 Apr 2020].

28 Bronfort G, Haas M, Evans RL, et al. Efficacy of spinal manipulation and mobilization for low back pain and neck pain: a systematic review and best evidence synthesis. Spine J 2004;4:335-56.

29 Bronfort G, Haas M, Evans R, et al. Effectiveness of manual therapies: the UK evidence report. Chiropr Osteopat 2010;18:3.

30 Coulter ID, Crawford C, Hurwitz EL, et al. Manipulation and mobilization for treating chronic low back pain: a systematic review and meta-analysis. Spine J 2018;18:866-79.

31 Nolet PS, Côté P, Cassidy JD, et al. The association between self-reported cardiovascular disorders and troublesome neck pain: a population-based cohort study. J Manipulative Physiol Ther 2012;35:176-83.

32 Ritzwoller DP, Crounse L, Shetterly S, et al. The association of comorbidities, utilization and costs for patients identified with low back pain. BMC Musculoskelet Disord 2006;7:72.

33 Bath B, Trask C, McCrosky J, et al. A biopsychosocial profile of adult Canadians with and without chronic back disorders: a populationbased analysis of the 2009-2010 Canadian community health surveys. Biomed Res Int 2014;2014:1-11.

34 Gore M, Sadosky A, Stacey BR, et al. The burden of chronic low back pain: clinical comorbidities, treatment patterns, and health care costs in usual care settings. Spine 2012;37:E668-77.

35 Robertson D, Kumbhare D, Nolet P, et al. Associations between low back pain and depression and somatization in a Canadian emerging adult population. J Can Chiropr Assoc 2017;61:96-105.

36 Feingold D, Brill S, Goor-Aryeh I, et al. The association between severity of depression and prescription opioid misuse among chronic pain patients with and without anxiety: a cross-sectional study. $J$ Affect Disord 2018;235:293-302.

37 Han B, Compton WM, Blanco C, et al. Prescription opioid use, misuse, and use disorders in U.S. adults: 2015 national survey on drug use and health. Ann Intern Med 2017;167:293-301.

38 Lisi AJ, Corcoran KL, DeRycke EC, et al. Opioid use among veterans of recent wars receiving Veterans Affairs chiropractic care. Pain Med 2018;19:S54-60.

39 Carroll LJ, Hogg-Johnson S, van der Velde G, et al. Course and prognostic factors for neck pain in the general population: results of the bone and joint decade 2000-2010 Task force on neck pain and its associated disorders. Spine 2008;33:S75-82.

40 O'Cathain A, Murphy E, Nicholl J. The quality of mixed methods studies in health services research. $J$ Health Serv Res Policy 2008;13:92-8.
41 Creswell JW, Plano Clark VL. Designing and conducting mixed methods research. 3rd ed. Thousand Oaks, CA: Sage, 2018.

42 Vassar M, Holzmann M. The retrospective chart review: important methodological considerations. J Educ Eval Health Prof 2013;10:12.

43 Tashakkori A, Teddlie C. Handbook of mixed methods in social and behavioural research. Thousand Oaks, CA: Sage, 2003.

44 Greene JC, Caracelli VJ, Graham WF. Toward a conceptual framework for mixed-method evaluation designs. Educ Eval Policy Anal 1989;11:255-74.

45 Globe G, Farabaugh RJ, Hawk C, et al. Clinical practice guideline: chiropractic care for low back pain. J Manipulative Physiol Ther 2016;39:1-22.

46 Katz MH. Multivariable analysis: a primer for readers of medical research. Ann Intern Med 2003;138:644-50.

47 Chan KS, Fowles JB, Weiner JP. Review: electronic health records and the reliability and validity of quality measures: a review of the literature. Med Care Res Rev 2010;67:503-27.

48 Choi BC, Noseworthy AL. Classification, direction, and prevention of bias in epidemiologic research. J Occup Med 1992;34:265-71.

49 Kleinbaum DG, Kupper LL, Nizam A, et al. Applied regression analysis and other multivariable methods. 5th ed. Boston, MA Cengage Learning, 2014.

50 Dobson AJ. An introduction to generalized linear models. 2nd ed. Boca Raton, FL: Chapman \& Hall/CRC, 2002.

51 Vittinghoff E, Glidden DV, Shiboski SC. Regression methods in biostatistics: linear, logistic, survival, and repeated measures models. 2nd ed. New York, NY: Springer, 2012.

52 Pan W. Akaike's information criterion in generalized estimating equations. Biometrics 2001:57:120-5.

53 Collins KMT, Onwuegbuzie AJ, Jiao QG. A mixed methods investigation of mixed methods sampling designs in social and health science research. J Mix Methods Res 2007;1:267-94.

54 Fetters MD, Curry LA, Creswell JW. Achieving integration in mixed methods designs-principles and practices. Health Serv Res 2013;48:2134-56.

55 Bradshaw C, Atkinson S, Doody O. Employing a qualitative description approach in health care research. Glob Qual Nurs Res 2017;4:233339361774228

56 Darnall BD, Ziadni MS, Stieg RL, et al. Patient-centered prescription opioid tapering in community outpatients with chronic pain. JAMA Intern Med 2018;178:707-8.

57 Maiers M, Hondras MA, Salsbury SA, et al. What do patients value about spinal manipulation and home exercise for back-related leg pain? A qualitative study within a controlled clinical trial. Man Ther 2016;26:183-91. 